\title{
Succinate homeostasis protects against lithogenesis and hypertension
}

Kidney stone formation is associated with hypertension but the underlying mechanism is unclear. Now, Ehud Ohana and colleagues report that a succinate homeostatic pathway provides a potential link between calcium oxalate $(\mathrm{CaOx})$ lithogenesis and increased blood pressure.

"We previously identified a metabolic pathway that regulates citrate and oxalate transport via the citrate and succinate transporter $\mathrm{NaDC} 1$ and the oxalate transporter SLC26A6," says Ohana. "These transporters form a complex and SLC26A6 inhibits NaDC1 activity. As this mechanism also governs transepithelial succinate transport, we hypothesized that SLC26A6 ${ }^{-/-}$mice, which develop $\mathrm{CaOx}$ stones, hyperoxaluria and hypocitraturia, would also have impaired succinate homeostasis."

The researchers found that in addition to elevated serum succinate levels, SLC26A6 $6^{-1-}$ mice have increased plasma renin levels and show activity-dependent hypertension. These findings were consistent with a previous report that succinate might contribute to blood pressure regulation by inducing renin release in the juxtaglomerular apparatus. The researchers show that luminal succinate sensing via the succinate receptor SUCNR1 regulates and orchestrates transepithelial succinate transport by inducing the translocation of a scaffolding protein, IRBIT, which interacts with the NaDC1-SLC26A6 complex and inhibits succinate transport by $\mathrm{NaDC} 1$.

"Our findings suggest that the molecular mechanism of succinate-citrate-oxalate homeostasis that we have delineated protects against kidney stone formation and controls blood pressure," concludes Ohana. "Succinate and citrate signalling and transport mechanisms should be considered potential therapeutic targets for the treatment of hypertension and urolithiasis."

Ellen F. Carney

ORIGINAL ARTICLE Khamaysi, A. et al. Systemic succinate homeostasis and local succinate signalling affect blood pressure and modify risks for calcium oxalate lithogenesis. J.Am. Soc. Nephrol. https://doi.org/10.1681/ASN.2018030277 (2019)

\section{SEPSIS}

\section{ABCF1 switches off inflammation in sepsis}

The mechanisms that regulate the transition between systemic inflammatory response syndrome (SIRS) and the endotoxin tolerance (ET) phase in sepsis are not yet clear. Now, Wilfred Jefferies and colleagues report that the ATP-binding cassette gene ABCF1 controls this transition by regulating innate immune responses in macrophages.

The researchers previously conducted a gene expression screen and identified ABCF1 as a potential regulator of immunity and inflammation. In their recent study, they show that $\mathrm{ABCF} 1$ is an E2 ubiquitin-conjugating enzyme that regulates macrophage polarization from the pro-inflammatory M1 to the anti-inflammatory M2 phenotype by promoting TLR4 endocytosis and activation of TRIF-dependent signalling. In a murine model of sepsis, ABCF1 haploinsufficiency led to increased expression of pro-inflammatory cytokines, failure to transition from SIRS to the ET phase, renal circulatory failure and increased mortality.

"During SIRS, the acute inflammatory response caused by pro-inflammatory cytokines damages the host tissue, which ultimately leads to death," explains Jefferies. "Histological analysis of the ABCF1-deficient mice in the SIRS phase revealed widespread dilation and congestion of small vessels in the kidneys as well as a sludging effect of red blood cells being compacted together in these vessels. This form of vasculature dilation and congestion on a systemic scale results in a critical drop in blood pressure, which leads to insufficient delivery of blood and oxygen to central organs, including the brain and heart."

The researchers conclude that ABCF1 modulates sepsis mortality by promoting transition to the ER phase, thereby repressing hypotension-induced renal circulatory failure. "As ABCF1 switches off pro-inflammatory pathways, our research may provide a map to explore new treatments for chronic and acute inflammatory diseases as well as autoimmune disorders," says Jefferies.

Ellen F. Carney

ORIGINAL ARTICLE Arora, H. et al. The ATP-binding cassette gene ABCF1 functions as an E2 ubiquitin-conjugating enzyme controlling macrophage polarization to dampen lethal septic shock. Immunity 50, 418-431 (2019)
ORGANOGENESIS

\section{Growing mouse kidneys in rats}

Interspecific blastocyst complementation is a potential strategy for growing human kidneys in animal hosts. Masumi Hirabayashi and colleagues now provide evidence that this approach is viable by using it to grow mouse kidneys in rats.

"The process of interspecific blastocyst complementation includes knocking out a gene responsible for renal development in the host animal, maintaining heterogeneous mutant lines to produce homozygous mutant blastocysts, and injecting these blastocysts with exogenous pluripotent stem cells that generate a kidney in the developmental niche," explains Hirabayashi.

Here, the researchers used CRISPR-Cas9 in rats to knock out Sall1, which encodes a protein that is essential for ureteric bud invasion in kidney development in mice. Like Sall1 ${ }^{-/-}$mice, Sall $1^{-/}$rats were anephric and thus a suitable host for generating mouse kidneys. Mouse embryonic stem cells were injected into blastocysts from intercrossed Sall $1^{+/-}$rats and transferred into pseudopregnant rat uteri. Of the 18 Sall1 neonates generated, 12 had a pair of kidneys derived from mouse embryonic stem cells. Furthermore, at postnatal day 0 (P0) there was patency between the ureter and the bladder in some mouse kidneys grown in rats, indicating their potential to excrete urine.

On previous work, Hirabayashi notes that "our initial attempts to grow rat kidneys in mice proved unsuccessful, as rat stem cells did not readily differentiate into the two main types of cells needed for kidney formation". By contrast, at P0 in mouse kidneys grown in rats, all the renal components that derive from metanephric mesenchyme were made entirely from mouse embryonic stem cells. However, the collecting ducts, glomerular capillaries and cortical blood vessels comprised a mixture of mouse and rat cells.

"Next we must regenerate blood vessels and collecting ducts in the kidneys with embryonic stem cells using the same blastocyst complementation strategy," says Hirabayashi. "Then, we will apply interspecific blastocyst complementation to large domestic species."

Katharine H. Wrighton

ORIGINAL ARTICLE Goto, T. et al. Generation of pluripotent stem cell-derived mouse kidneys in Sall1-targeted anephric rats. Nat. Commun. 10, 451 (2019) 